\title{
ENHANCED LOW POWER, FAST AND AREA EFFICIENT CARRY SELECT ADDER
}

\author{
Prajwal $\mathbf{S}^{\mathbf{1}}$ \\ ${ }^{I}$ MTech (Digital Electronics and Communication), MSRIT, Bangalore, India
}

\begin{abstract}
Adder being the basic hardware block of any arithmetic operation, the major constraint in the field of signal processors, data processors to perform any operations is highly dependent on the adder performance of the circuit. The gate level implementation of the carry select adder (CSLA) and modified carry select adder has significantly reduced the area and power consumption which replaced the ripple carry adder (RCA) used in modified CSLA with MUX based Full Adder (MUX-FA) block has further reduced the power consumption by efficiently utilizing the area with faster performance.
\end{abstract}

Keywords-Application-specific integrated circuit (ASIC), area efficient, MUX-FA, CSLA, low power.

\section{INTRODUCTION}

Switching speed with low power consumption is the major area needed to be concentrated in modern trends of signal processing, data processing and VLSI applications. To perform any kind of signal and data processing operation, fast arithmetic functions are to be calculated with higher speed but non-degrading functionality. With the advancements in the technology the factors to be taken care during the hardware designs are frequency or speed of operation, power consumption, area utilization, circuit complexity, portability, robustness etc. Thus while designing a modern high performance processing element the optimization or best utilization of the above mention factors are to be considered.

In any modern processing element the digital adder block is a basic block which ensures the high- speed performance to a large extend. The drawback of a simple ripple carry adder (RCA) is associated with its propagation of carry bit which is highly overcome by the implementation of high-speed, area efficient carry select adder (CSLA)[1]. The traditional CSLA independently generates multiple carry and then with the selected carry generates the sum which reduces the carry propagation delay of the RCA. The CSLA has been modified further by reducing the area and power consumption [2] to [4]. The implementation of square- root CSLA (SQRT CSLA) [5] \& [6] is modified with the usage of binary to excess-1 converter (BEC) instead of RCA [7] which claims an improved performance.

In this paper the usage of an element ripple carry adder is eliminated and instead a MUX-FA based arithmetic adder block is used which proves to be logically stronger with a reduced propagation delay in comparison to the other existing logic styles for full-adders such as standard CMOS, complementary pass transistor logic (CPL), double pass transistor logic (DPL), swing restored CPL (SR-CPL)
[8].

\section{MUX -FA BLOCK}

The main idea of this paper is to replace RCA from modified CSLA by a MUX-FA unit for improved performance. The MUX being a faster hardware than direct adder block mainly improvises the performance in terms of delay, area and power consumption. The truth table of full adder is studied and shown in table.1 with respect to carry input (Cin).

Table.1: Truth table of FA with respect to Cin.

\begin{tabular}{|c|c|c|c|c|c|c|}
\hline Cin & $\mathrm{A}$ & B & Sum & & Carry & \\
\hline (0) & 0 & 0 & $\mathbf{0}$ & \multirow{4}{*}{$\begin{array}{l}\mathbf{X} \\
\mathbf{O} \\
\mathbf{R}\end{array}$} & $\mathbf{0}$ & \multirow{4}{*}{$\begin{array}{l}\mathbf{A} \\
\mathbf{N} \\
\mathbf{D}\end{array}$} \\
\hline 0 & 0 & 1 & 1 & & 0 & \\
\hline 0 & 1 & 0 & 1 & & $\mathbf{0}$ & \\
\hline 0 & 1 & 1 & 0 & & 1 & \\
\hline 1 & 0 & 0 & 1 & \multirow{4}{*}{$\begin{array}{l}\mathbf{X} \\
\mathbf{N} \\
\mathbf{O} \\
\mathbf{R}\end{array}$} & $\mathbf{0}$ & \multirow{4}{*}{\begin{tabular}{|l}
$\mathbf{O}$ \\
$\mathbf{R}$
\end{tabular}} \\
\hline 1 & 0 & 1 & 0 & & 1 & \\
\hline 1 & 1 & 0 & $\mathbf{0}$ & & 1 & \\
\hline 1 & 1 & 1 & 1 & & 1 & \\
\hline
\end{tabular}

The MUX-FA (1-bit) implementation is shown in figure.1

The BEC based carry select addition happens by following the algorithm below: 


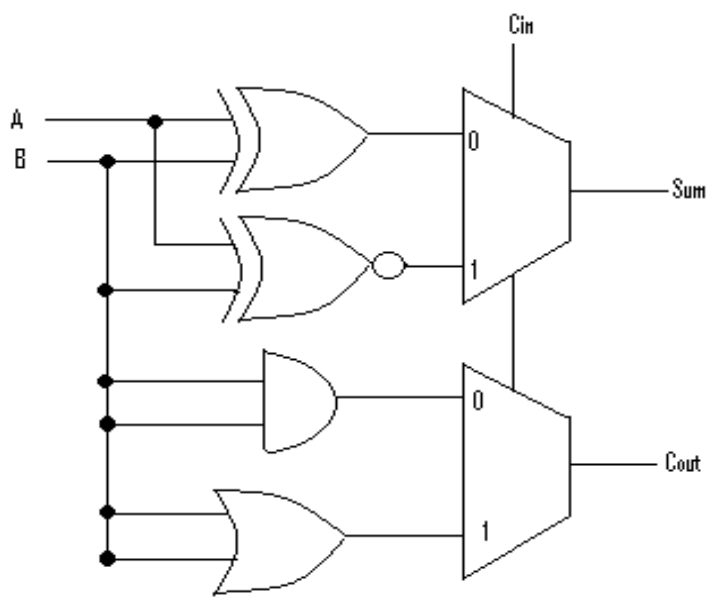

Fig 1: 1-bit MUX-FA Implementation

\section{BEC \& BEC BASED CSLA}

Binary to excess- 1 converter is used to perform the addition task faster whenever the carry input is ' 1 '. The CSLA for Cin=' 1 ' is obtained by the implementation of BEC. For a 4bit BEC it takes the four bits as input and the output is four bits excess to one as shown in figure.2.


Fig 2: 4-bit BEC

Table.3: Delay, area, power, PDP \& ADP calculations of different modules.

\begin{tabular}{|l|l|l|l|l|l|l|l|}
\hline $\begin{array}{l}\text { With reference from } \\
\text { table.4 the usage of MUX- } \\
\text { FA \& BEC based CSLA }\end{array}$ & & $\begin{array}{l}\text { AREA } \\
\left.\mu \mathrm{m}^{2}\right)\end{array}$ & $\begin{array}{l}\text { POWER(nW) } \\
\begin{array}{l}\text { Leakage } \\
\text { power }\end{array}\end{array}$ & $\begin{array}{l}\text { Switching } \\
\text { Power }\end{array}$ & $\begin{array}{l}\text { Total } \\
\text { power }\end{array}$ & $\begin{array}{l}\text { Power- } \\
\text { Delay product(nW- } \\
\text { ns) }\end{array}$ & $\begin{array}{l}\text { Area-Delay } \\
\text { product }\left(\mu \mathrm{m}^{2}-\right. \\
\text { ns) }\end{array}$ \\
\hline 1-bit RCA/FA & 2.164 & 30 & 16.919 & 676.008 & 692.927 & 1499.49 & 64.92 \\
\hline 1-bit MUX-FA & 1.154 & 42 & 20.472 & 716.274 & 736.746 & 850.204 & 48.468 \\
\hline 4-bit BEC & 2.145 & 39 & 23.192 & 814.597 & 837.789 & 1797.057 & 83.655 \\
\hline
\end{tabular}




\begin{tabular}{|l|l|l|l|l|l|l|l|}
\hline 4-bit RCA with BEC & 1.588 & 123 & 71.783 & 3077.694 & 3149.477 & 5001.369 & 195.324 \\
\hline $\begin{array}{l}\text { 4-bit MUX- FA with BEC } \\
\text { (proposed model) }\end{array}$ & 1.138 & 126 & 61.417 & 2428.017 & 2489.434 & 2584.032 & 130.788 \\
\hline
\end{tabular}

\section{PROPOSED DESIGN}

The proposed design replaces the regular RCA for calculating the higher bit summation by MUX-FA. This MUX-FA being faster and low power consumable block improves the overall performance of the circuit. In the proposed design, as shown in figure.3, the first bit is calculated using a direct one bit MUX-FA block to calculate SUM0. The next higher order bits are calculated in two parts i.e. for Cin='0' where it's a simple carry addition using MUX-FA blocks \& the other one for Cin='1' using BEC. Both the output sets from MUX-FA blocks and BEC is given as the input to 8:4MUX and the final output of sum and carry are taken from the MUX output. Figure 3 depicts the 4-bit implementation of MUX-FA \& BEC based CSLA which can be extended for higher bits.

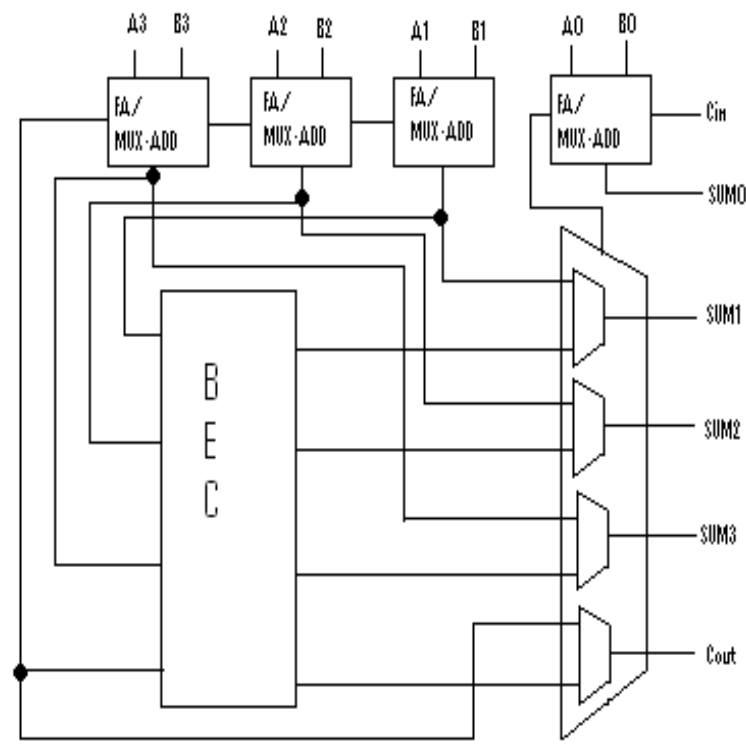

Fig 3: 4-bit RCA/MUX-FA \& BEC based CSLA

\section{SIMULATION}

The design proposed in this paper has been developed using Verilog - HDL and synthesized using Cadence RTL. Simulation is done using Cadence Simvision. Table. 3 shows the simulation results for the different modules used in this paper regarding delay, power consumption, area, Power Delay Product (PDP) \& Area-Delay Product (ADP). The total power is the sum of leakage power \& switching power where as the area indicates the total cell area of the design.

\section{CONCLUSIONS}

With reference from table. 4 the usage of MUX-FA \& BEC based CSLA has $48.3 \%$ of improvement in PDP and $33.04 \%$ of improvement in ADP in comparison to modified CSLA (4bit, can be extended for higher bit size) and delay reduces by $34.63 \%$. Thus with maintaining optimization \& proper functionality MUX-FA \& BEC based CSLA claims to be highly efficient. The similar work can be extended for higher order bits i.e. for higher word size and implemented to use in modern processors.

\section{REFERENCES}

[1]. O.J. Bedrij, "Carry-select adder,"IRE Tran's. Electron. Comput., pp.340-344, 1962.

[2]. B. Ramkumar, H. M. Kittur, and P. M. Kannan, "ASIC implementation of modified faster carry save adder,"Eur. J. Sci. Res., vol. 42, no. 1, pp. 53-58, 2010.

[3]. T. Y. Ceiang and M. J. Hsiao, "Carry-select adder using single ripple carry adder,"Electron. Lett., vol. 34, no. 22, pp. 2101-2103, Oct. 1998. .

[4]. J. M. Rabaey, Digtal Integrated Circuits-A Design Perspective. Upper Saddle River, NJ: Prentice-Hall, 2001.

[5]. Y. He, C. H. Chang, and J. Gu, "An area efficient 64bit square root carry-select adder for low power applications," inProc. IEEE Int. Symp. Circuits Syst., 2005, vol. 4, pp. 4082-4085.

[6]. B.Ramkumar and Harish M Kittur, "Low power area efficient Carry select adder", IEEE transaction on VLSI Systems, Vol: 20, No.2, February 2012, pp. 371-375. 